\title{
LEAF ADAPTABILITY OF TWO NON-TIMBER FOREST SPECIES Caryota urens L. AND Elettaria cardamomum VAR. MAJOR PLANTED \\ IN THE Pinus caribaea ENRICHMENT BUFFER ZONE IN SINHARAJA MAB RESERVE, SRI LANKA
}

\author{
H M V T Welagama, K U Tennakoon, C V S Gunatilleke, \\ I A U N Gunatilleke and R M M Bandara \\ Department of Botany, University of Peradeniya
}

Caryota urens L. and Elettaria cardamomum var. major are indigenous non-timber species which are used as market or sustainable goods and services for human or industrial consumption. Both species thrive well in disturbed forest areas in the southwestern part of Sri Lanka. Being understorey species, the growth of these species are affected by sun light which is highly variable and often limiting resource in the forest.

In a Pinus enrichment study established in 1991, leaf anatomical adaptability of these two species after 12 years of initial establishment under the Pinus buffer zone of the Sinharaja MAB reserve was investigated. Different light levels in the study area were created by thinning Pinus trees at different densities.

Lower epidermis of $C$. urens showed highest $(7.75 \%)$ and lowest $(6.35 \%)$ stomatal indices in 2 pine rows removed treatment $(2 R)$ and 5 pine rows removed treatment $(5 R)$ respectively. Corresponding values for $E$. cardamomum were $6.00 \%$ (highest) in the closed canopy control (CU) and $5.22 \%$ (lowest) in 1 pine row removed treatment (IR).

The highest thickness of $C$. urens leaf cuticle $(0.26 \mu \pm 0.02)$, leaf blade $(22.27 \mu \pm 2.14)$, upper epidermis $(1.75 \mu \pm 0.10)$, lower epidermis $(1.83 \mu \pm 0.06)$, palisade layer $(6.25 \mu$ $\pm 0.87)$ and spongy mesophyll layer $(11.74 \mu \pm 1.30)$ were observed in $5 R$ treatment that received relatively high light levels than other treatments.

E. cardamomum leaves showed highest thickness of leaf blade $(22.43 \mu \pm 0.88)$, lower cpidermal layer $(2.12 \mu \pm 0.11)$, spongy mesophyll layer $(10.32 \mu \pm 0.57)$ in 3 pine rows removed treatment (3R). The highest cuticle thickness $(0.23 \mu \pm 0.01)$ was recordered in 3 pine rows under planting treatment $(3 \mathrm{U})$ while $1 \mathrm{R}$ treatment showed highest upper epidermal layer thickness of $2.21 \mu \pm 0.15$. Palisade layer was highest $(4.74 \mu \pm 0.55)$ in CU.

Results revealed that $C$. urens shows leaf adaptability to grow under relatively high sun light levels (5R treatment) while $E$. cardamomum shows most of the leaf adaptability characters to grow under moderate light levels (3R treatment).

The knowledge gained by this study is useful to recommend the optimum light requirements in terms of leaf anatomical traits of the study species.

Proceedings of the Ninth Annual Forestry and Environment Symposium 2003 of the Department of Forestry and Emvironmental Science, University of Sri Jayewardenepura. Sri Lanka 